\title{
Long-Term Prediction of Soybean Rust Entry into the Continental United States
}

\author{
Z. Pan, Department of Earth and Atmospheric Sciences, St. Louis University, St. Louis, MO 63103; X. B. Yang, \\ Department of Plant Pathology, Iowa State University, Ames 50011; S. Pivonia, Arava R\&D, Sapir, P.O. Box. Arava, \\ 86825 ISRAEL; L. Xue and R. Pasken, Department of Earth and Atmospheric Sciences, St. Louis University, St. \\ Louis, MO 63103; and J. Roads, Scripps Institution of Oceanography, UCSD, 0224, La Jolla, CA 92093
}

\begin{abstract}
Pan, Z., Yang, X. B., Pivonia, S., Xue, L., Pasken, R., and Roads, J. 2006. Long-term prediction of soybean rust entry into the continental United States. Plant Dis. 90:840-846.

This special report demonstrates the feasibility of long-term prediction of intercontinental dispersal of Phakopsora pachyrhizi spores, the causal agent of the devastating Asian soybean rust (SBR) that invaded the continental United States in 2004. The climate-dispersion integrated model system used for the prediction is the combination of the particle transport and dispersion model (HYSPLIT_4) with the regional climate prediction model (MM5). The integrated model system predicts the trajectory and concentration of $P$. pachyrhizi spores based on threedimensional wind advection and turbulent transport while incorporating simple viability criteria for aerial spores. The weather input of the model system is from a seasonal global climate prediction. The spore source strength and distribution were estimated from detected SBR disease severity and spread. The model system was applied to the known P. pachyrhizi spore dispersal between and within continents while focusing on the disease entry into the United States. Prediction validation using confirmed disease activity demonstrated that the model predicted the 2004 U.S. entry months in advance and reasonably forecast disease spread from the south coast states in the 2005 growing season. The model also simulated the dispersal from Africa to South America and from southern South America to Columbia across the equator. These validations indicate that the integrated model system, when furnished with detailed source distribution, can be a useful tool for P. pachyrhizi and possibly other airborne pathogen prediction.
\end{abstract}

Additional keywords: climate model, hurricane

Phakopsora pachyrhizi, the causal agent of Asian soybean rust (SBR), was confirmed on 10 November 2004 in Louisiana (27) and subsequently in several other states along the Gulf Coast (Fig. 1). This was the first time the $P$. pachyrhizi infection was detected in the continental United States, and it happened about 3 months following its detection on 4 August 2004 in Cali (at $3.7^{\circ} \mathrm{N}, 76^{\circ} \mathrm{W}$ ), Columbia (16).

Asian soybean rust was first recorded in Japan in 1903 and had caused severe yield losses in Asia for decades. The disease was identified for the first time in the Western Hemisphere in Hawaii in 1994 (9). In 1996, the disease was first reported in Uganda, and by 2001 it had spread throughout much of Africa (21,22 [and references therein]). In 2001, soybean rust was found in South America, and it has since spread throughout the soybean grow-

Corresponding author: Zaitao Pan

E-mail: panz@eas.slu.edu

Accepted for publication 13 February 2006.

DOI: 10.1094/PD-90-0840

(C) 2006 The American Phytopathological Society ing areas of Brazil, Paraguay, Argentina, and Bolivia. Threats of soybean rust to the United States have been assessed in terms of yield loss (28) or economic damage $(8,10)$. It is not unusual for the SBR to reduce yields by half. Severe outbreaks in the last few years in South America have increased concern about the spread of the disease to the North American soybean producing regions.

Scientists and policy makers have been prepared for the arrival of $P$. pachyrhizi on U.S. soils for several years. As such, researchers such as the USDA/APHIS group have attempted to predict the arrival of the disease $(6,7)$. While the modeling efforts in APHIS focus on short-term day-to-day forecasts, our model system emphasizes the long-term prediction of spore movements both among continents and within the United States. The long-term prediction of rust spore movements is crucial for decision making in disease control and general farm planning. To the authors' knowledge, there are no other long-term, dynamic, and physical process-based models that can predict future spore movement a month in advance.

Like long-term prediction of weather and climate, there are two general ap- proaches used in the prediction of longdistance transport (LDT) of P. pachyrhizi spores. The first one is statistical and is based upon climatological atmospheric movements. The future movements of spores are inferred from statistical trajectory or concentration distribution established using past history (7). Although this approach can provide the overall climatological map of spore movements, it is unlikely to predict extreme situations for a particular year, which is often the case with spore dispersal $(13,14)$. The second approach is a predictive modeling approach where dynamic models are used to predict the atmospheric parcel and thus airborne spore movements based on mathematical governing equations $(6,12$, 18-20). Since the built-in model equations describe general conditions including extremes, the dynamic approach, in principle, is more likely to capture abnormal weather events. The latter approach has been used in seasonal climate prediction $(23,24)$, but to the authors' knowledge the dynamic approach has not been used for long-term spore prediction.

In terms of the spore dispersal model itself, there are also two general methods: kinematic and dynamic. In the former, air parcels or spores move kinematically with certain prespecified spatial spread (e.g., IAMS parcel-box model [6,7]). In the latter, the spore dispersal is dynamically dictated by basic laws such as momentum and energy conservation (e.g., HYSPLIT_4 [3]). Our models are dynamic in both weather prediction and spore dispersal, as described in the Materials and Methods section below.

The objectives of this study are to (i) describe the model system, (ii) test the feasibility of dynamic, long-term prediction of rust spore dispersal by applying the model to recent known rust LDT cases, and (iii) examine the mechanisms by which rust entered the continental United States, including anomalous hurricane activity.

\section{MATERIALS AND METHODS}

The integrated model system. The integrated rust prediction model system consists of a global and a regional atmospheric prediction model and a particle transport and diffusion model (Fig. 2). The dynamic 
prediction core of the model system is the mesoscale model MM5 developed jointly at Penn State University (PSU) and National Center for Atmospheric Research (NCAR) (4,5). The MM5 solves the full suite of prognostic primitive equations for three-dimensional winds, temperature, clouds, and radiation. The model includes a number of options for boundary layer schemes, clouds and precipitation treatments, and radiative transfer. It is a widely used regional model and has shown high forecasting skills in many parts of the world including South and Central America (1). The MM5 software including all source codes is freely provided and supported by NCAR $(4,5)$. In our application, the model domain is configured to cover $127 \times 97$ grid points with a horizontal resolution of $120 \mathrm{~km}$ centered at $\left(1.0^{\circ} \mathrm{N}\right.$, $\left.76.0^{\circ} \mathrm{W}\right)$. An inner nested domain covers Central America including the southeastern United States with a resolution of $40 \mathrm{~km}$.

Although the region of interest for this study is the Americas, our model requires lateral boundary conditions from a global forecast of atmospheric conditions because MM5 is a limited-area model. The current national forecast centers produce only monthly, or at most, daily variables on seasonal range. However, the MM5 requires subdaily, typically at 6-h intervals, variables to drive it as lateral boundary conditions. The Experimental Climate Prediction Center (ECPC) of Scripps Institution of Oceanography now routinely makes seasonal prediction of climate on a global scale $(23,24)$. The 6-hourly threedimensional winds, temperature, pressure, and moisture on $1.875^{\circ} \times 1.875^{\circ}$ grids from ECPC were imposed on the MM5's 11-grid-point nudging zone where the MM5 variables were nudged to ECPC variables every time step. The MM5 output of atmospheric conditions is then fed into the spore dispersion model.

The dispersion (transport and diffusion) model used in our integrated model system is the HYSPLIT_4 model developed at NOAA Air Resource Lab. The model, described by Draxler and Hess (3), uses a hybrid Eulerian-Lagrangian approach to calculate the trajectories and concentrations of spores or particles in the air and on the ground. The input of HYSPLT_4 from MM5 output includes 6-hourly threedimensional winds, temperature, moisture, clouds, solar radiation, and precipitation. The output of HYSPLIT_4 is threedimensional time-varying spore concentration in the air, surface deposition, and trajectories.

Source strength. In the model, the rust spores are released from inside canopies, then carried out of canopies, and finally lifted above the atmospheric boundary layer by convection. The spore releasing rate and lifting efficiency are largely unknown for $P$. pachyrhizi spores; so in this study, the source strength is assumed to be

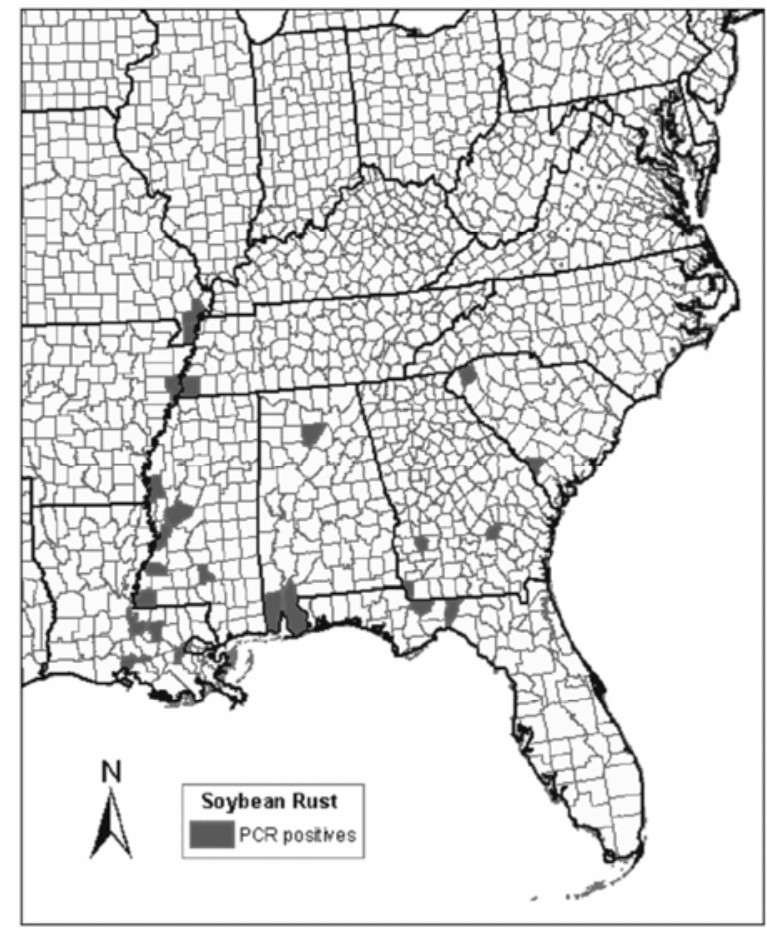

Fig. 1. Sites (in darkened region) where Asian soybean rust (Phakopsora pachyrhizi) was confirmed by USDA/APHIS in the continental United States during November 2004. The map was adapted from the Department of Crop Sciences, University of Illinois at Urbana-Champaign (available online.)

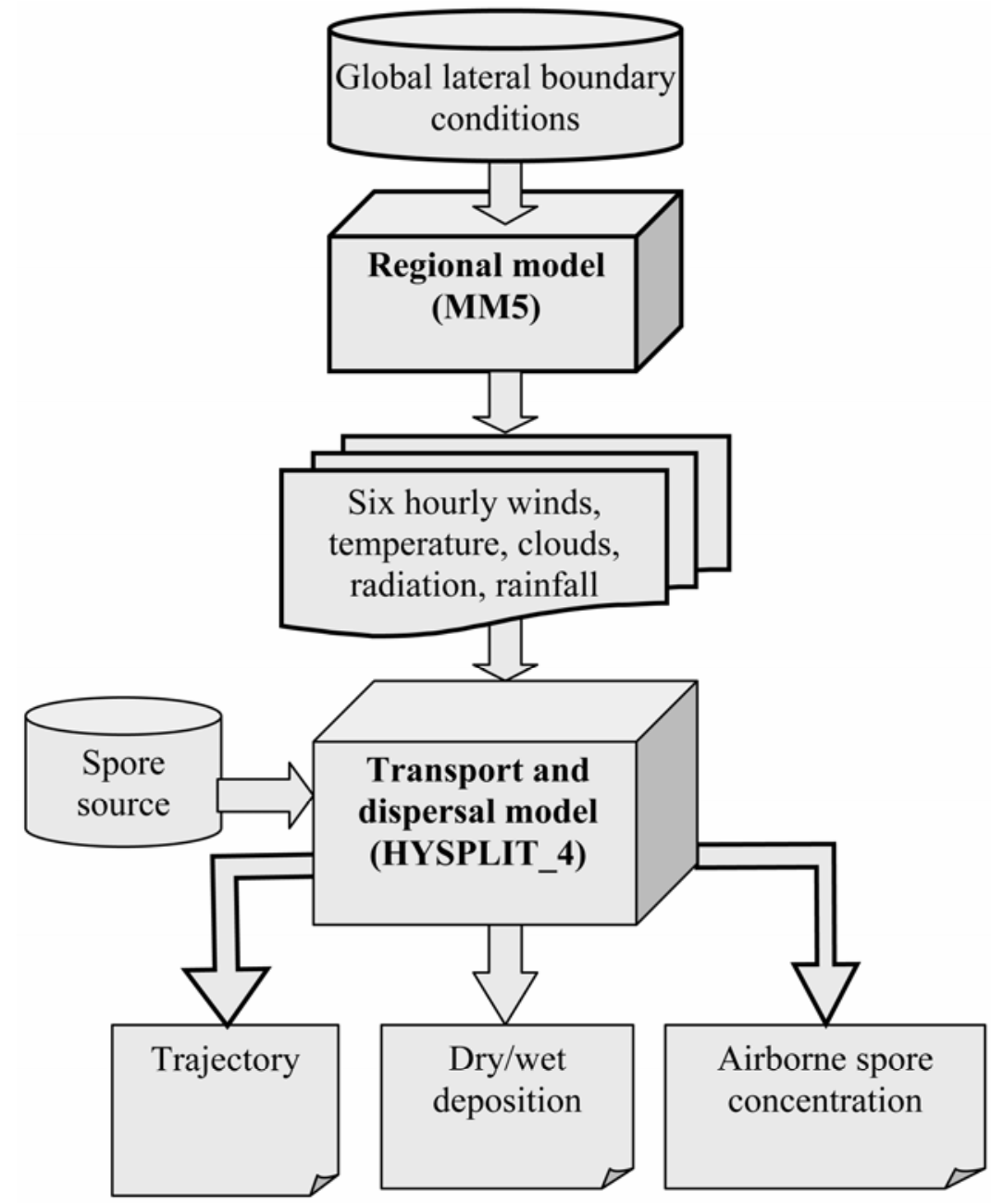

Fig. 2. Schematic diagram of the soybean rust model prediction system showing component models and data flow structure. 
$10^{9}$ spores per hectare per day based on estimates of plant density, disease severity and incidence, and escape efficiency (from canopy) in a similar way to that described by Isard et al. (6). Since the exact strength and locations of $P$. pachyrhizi spore sources in the Cali region, where the rust was first found in the northern hemisphere in the Americas, are impossible to determine, we experimented with low and high releasing rate scenarios while using evenly distributed sources in the $2^{\circ} \times 2^{\circ}$ rectangle centered in Cali (Fig. 3). We use 9 and 90 releasing points for the low and high rate scenarios, respectively. Once the spores are lifted into the free atmosphere, strong winds transport them horizontally.

Transport and dispersion. Advection (transport) and diffusion (dispersion) calculations are made in a Lagrangian framework while concentrations are calculated on a fixed grid. HYSPLIT_4 uses a puffparticle combined approach by assuming a puff distribution in the horizontal and particle dispersion in the vertical direction. The advection of both particles and puffs is computed from three-dimensional velocity vectors. The particle dispersion is determined from the turbulent velocity components, while puff dispersion is treated differently depending on the puff size. Additional detail can be found in the HYSPLIT_4 model descriptions $(3,15)$.

Dry and wet deposition. Dry deposition speed is computed as the gravitational settling velocity. Particle wet removal is computed from a scavenging ratio within clouds and below clouds. For computa-

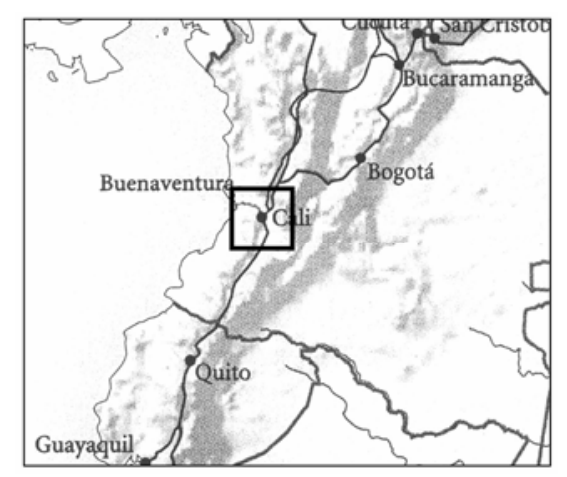

Fig. 3. Asian soybean rust was reported five degrees north of the equator in Cali, Columbia, in August of 2004. The rectangle indicates the approximate spore source region used in this study. tional simplicity, the total deposition from both dry and wet removal processes is expressed in terms of (reciprocal) time constants. The dry deposition depends on deposition velocity and spore layer depth; in-cloud wet removal of particles is determined by wet deposition rate and precipitation rate; and below-cloud wet removal of particles is assumed to be constant, independent of precipitation.

Spore viability. Airborne $P$. pachyrhizi spore survivability depends on atmospheric conditions such as temperature, solar (UV-B) radiation, and natural mortality. Currently, the model considers the radiation exposure and limited life span reflecting the natural mortality while assuming that temperature is not a limiting factor as the model was run primarily for tropical climate. Since little is known about quantitative $P$. pachyrhizi spore viability criteria, a step function was used: spores are assumed nonviable if they are exposed to a cumulative 60 megajoules (MJ) of total solar radiation per square meter (about two sunny days of radiation). This value is based on studies of other similar spores (25) and in rough agreement with other soybean rust studies (6). The incoming solar radiation is computed in MM5 and passed into HYSPLIT_4. The other viability constraint is the natural mortality. Rust spores are assumed nonviable after being airborne for more than 10 days.

Ensemble trajectory frequency. We ran HYSPLIT_4 in concentration and trajectory modes. Concentration distribution is typically widespread so that it masks detailed spatial variation, and thus it is difficult to accurately pinpoint spore locations. On the other hand, the trajectory mode produces only a single or a few isolated trajectories, which can easily miss spore activity. In this study, we use an ensemble trajectory frequency approach that, to a certain degree, combines advantages of both modes. This method is based on massive trajectories from a large number of sources over the whole prediction period, and thus it is unlikely to miss spore occurrences, and at the same time the accumulated trajectory frequency still keeps the sharpness of the concentrated trajectories. Because of the relative sharpness of the trajectory frequency distributions, this method can provide focused guidance for early disease detection efforts. The ensem- ble frequency measures how likely it is that spores exist at a particular location in a statistical sense. A high-frequency region represents preferable pathways of $P$. pachyrhizi spores.

Model prediction procedure. We applied the integrated model system as described above to $P$. pachyrhizi entry into the continental United States as well as the LDT from Africa to South America in 2001 and from southern South America to Columbia in 2004. In addition, during the 2005 growing season, the model system was run quasi-operationally once a week to predict the $P$. pachyrhizi spread within the United States. For each model prediction, first the global seasonal prediction output from the ECPC is processed according to the MM5 input format. Then MM5 is run to predict atmospheric movement over the domain of interest. Finally, the HYSPLIT_4 fed on MM5 output is run to produce the spore trajectories and concentration in hourly intervals over the entire forecast domain (Fig. 2). Graphics software accompanied with the models is used to depict the spore clouds' movements at various levels.

The period to be predicted for the U.S. entry case started on 7 August 2004, right after the first SBR detection in Cali (Fig. 3 ), Columbia, and lasted for 40 days. During the 40-day integration, the model used only information available on the starting date, i.e., the model was run purely predictively. The predicted periods for the other regions and years vary depending on individual cases.

\section{RESULTS}

Validation of long-term prediction of climate conditions. Table 1 lists mean forecast and hindcast errors and their ratio to variance (in parentheses). The values are computed over the entire domain for the 40-day period at $40 \mathrm{~m}, 1,000 \mathrm{~m}$, and 5,000 $\mathrm{m}$ above the ground. The domain-average errors in wind components in hindcast mode are $<0.3 \mathrm{~m} \mathrm{~s}^{-1}$ at the three levels. The error to variance ratio is $<10 \%$. As expected, forecast errors are larger than hindcast with wind errors of $1.3 \mathrm{~m} \mathrm{~s}^{-1}$ at the surface, which is $30 \%$ of variance. Typically, the LDT of spores is largely determined by upper-air movements, most likely close to the 1,000-m level (19). Thus, the larger wind errors near the surface may not have affected the spore trans-

Table 1. Domain-average model forecast and hindcast errors and ratios (in parentheses) of errors to observed variances averaged over the 40-day period starting 7 August 2004

\begin{tabular}{|c|c|c|c|c|c|c|}
\hline \multirow[b]{2}{*}{ Height (m) } & \multicolumn{3}{|c|}{ Hindcast } & \multicolumn{3}{|c|}{ Forecast } \\
\hline & $\begin{array}{c}\text { East-west }^{\mathrm{a}} \\
\text { wind speed (m/s) }\end{array}$ & $\begin{array}{c}\text { North-south } \\
\text { wind speed }(\mathrm{m} / \mathrm{s})\end{array}$ & Temperature $\left({ }^{\circ} \mathbf{C}\right)$ & $\begin{array}{c}\text { East-west } \\
\text { wind speed }(\mathbf{m} / \mathbf{s})\end{array}$ & $\begin{array}{c}\text { North-south } \\
\text { wind speed }(\mathrm{m} / \mathrm{s})\end{array}$ & Temperature $\left({ }^{\circ} \mathbf{C}\right)$ \\
\hline 5,000 & $0.2(6 \%)$ & $0.2(10 \%)$ & $0.3(3 \%)$ & $0.0(1 \%)$ & $-0.3(11 \%)$ & $-0.5(-5 \%)$ \\
\hline 1,000 & $-0.2(-5 \%)$ & $0.3(9 \%)$ & $-1.0(13 \%)$ & $-0.1(2 \%)$ & $-0.1(-3 \%)$ & $-1.8(-24 \%)$ \\
\hline 40 & $0.2(5 \%)$ & $0.0(1 \%)$ & $0.1(4 \%)$ & $1.3(30 \%)$ & $0.4(13 \%)$ & $-1.1(-31 \%)$ \\
\hline
\end{tabular}

a Wind is a vector defined by two perpendicular components: east-west and north-south. Positives winds flow toward the east and north, respectively. 
port as greatly. These meteorological errors are much smaller than those of disease biological uncertainty, much of which is not quantifiable.

Concentration prediction. Spore concentration was averaged over the 40-day period at $10 \mathrm{~m}$ above the ground, the standard height for observed surface winds. The largest spore concentration was dispersed west-northwestward to the Pacific Ocean (Fig. 4A), mainly following prevailing winds associated with the inter-tropical convergence zone (ITCZ) during the time. However, a relatively low concentration area extended toward the United States, covering the Gulf Coast states. It is interesting to see that in Louisiana (LA), where $P$. pachyrhizi infection was first detected, there was a local maximum center matching the first report of the disease in the United States (see Fig. 1).

At the 500-m height, where advection of flow was more dominant compared with that near the surface, the concentration shows primarily a west-northwest dispersal pattern (Fig. 4B). In contrast to that at the 10-m height, however, a distinctive secondary maximum concentration region branched out from the main spore concentration cloud. This branch reached the Gulf Coast states, apparently attributable to tropical storms. This secondary concentration maximum extending to a high altitude indicates a deep layer of the atmosphere loaded with concentrated $P$. pachyrhizi spores over those states. When rainfall occurs, these aerial spores can be easily washed to the surface.

Trajectory frequency prediction. The predicted spore trajectory frequency distribution indicated that large amounts of spores crossed southeastern Mexico and parts of the Gulf of Mexico and then finally reached the continental United States (Fig. 5A). High-frequency areas also covered southern and eastern Texas (TX) and LA, while moderate frequency areas covered Georgia (GA) and Florida (FL). Two major branches of spores survived the viability criteria when the higher releasing rate was used (Fig. 5B). The major branch was to the west-northwest associated with the ITCZ, and the secondary branch moved toward the north-northwest direction. This branch was associated with hurricane movements and further consisted of two distinct strands of spores that moved northward directly toward the United States. One started from the southern tip of the Gulf of Mexico along the eastern coast of Mexico and then finally reached LA. This pathway matches closely the detected locations of the rust shown in Figure 1. This strand also resembles the track of tropical storm Bonnie developed over the Gulf of Mexico during 9 to 12 August 2004, although the model-predicted track is somewhat west of that observed. The other strand directly extended from Cali to GA and FL. Its frequency was lower than the western one, in agreement with relatively less rust occurrence in these two
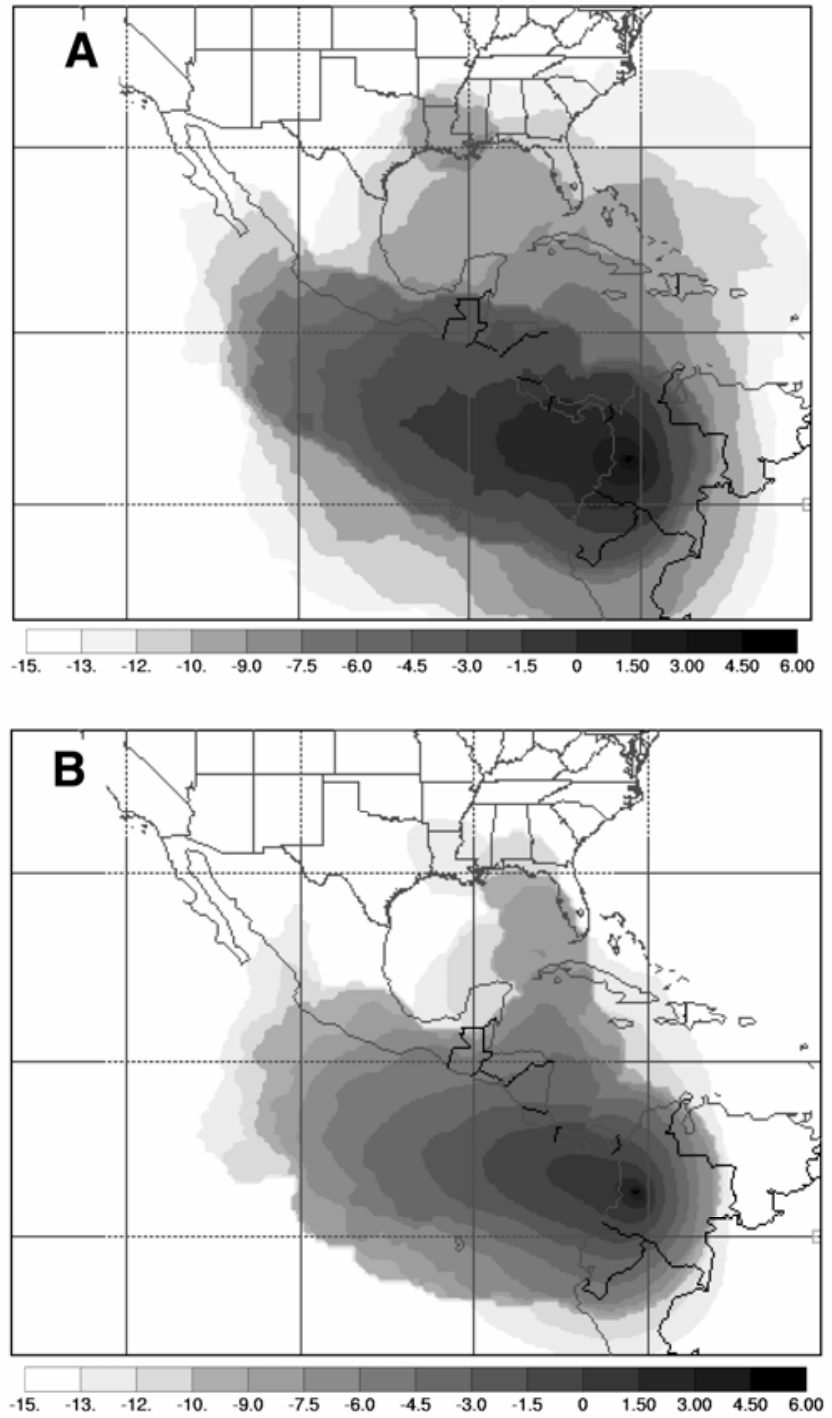

Fig. 4. The 40-day mean spore concentration predicted by the model showing the movement of spore clouds from Cali to the southern coast of the United States starting 7 August 2004. The unit is logarithmic scale (spore $\mathrm{m}^{-3}$ ). A, 10-m height aboveground. B, 500-m height aboveground.
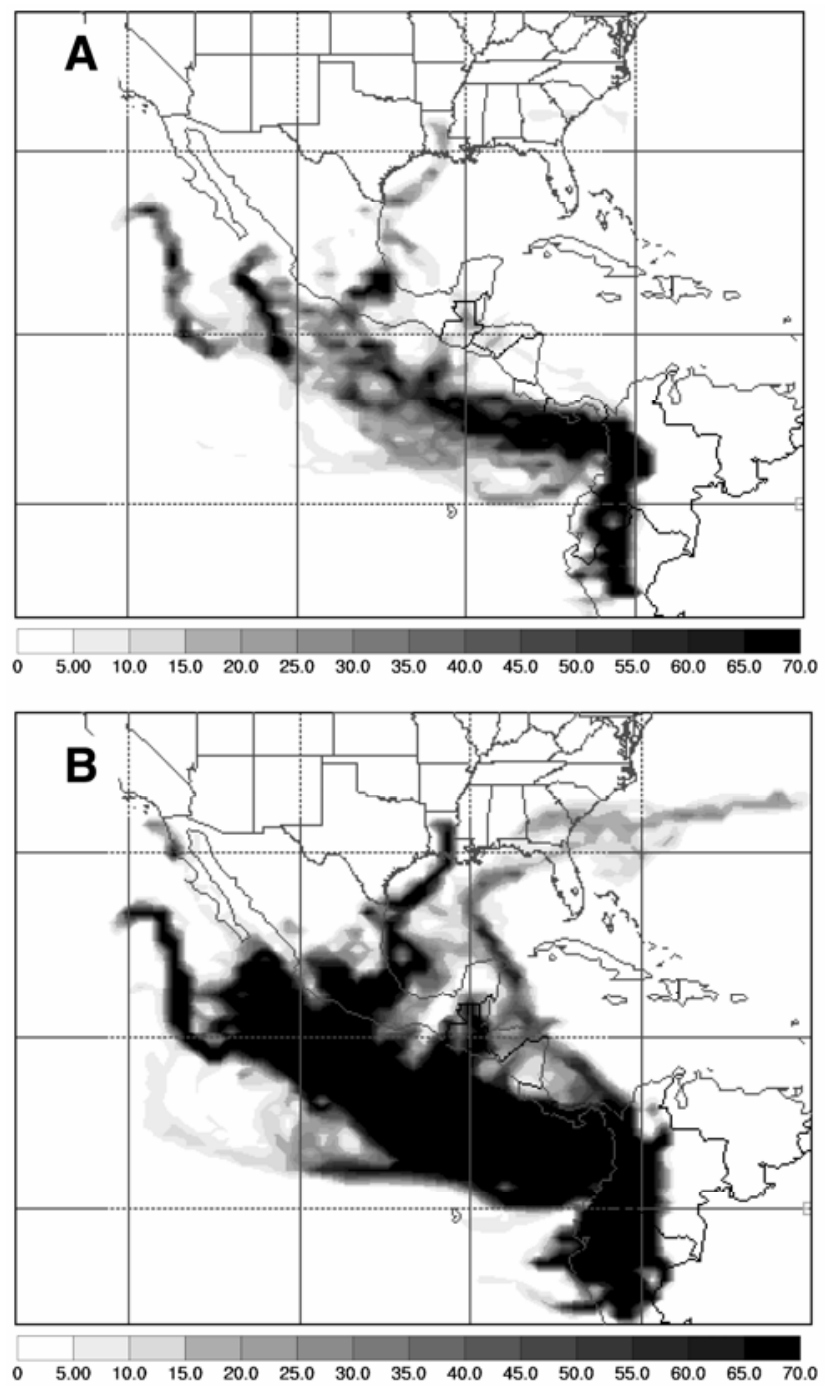

Fig. 5. Predicted trajectory frequency at 10-m height during the 40-day period starting 7 August 2004. High frequency represents preferred pathways. A, Low source intensity (9 source points). B, High source intensity (90 source points). The source is located at Cali, Columbia, indicated by the dark rectangle in Figure 3. 
states (GA and FL) compared with LA and Mississippi. This strand again was associated with storm cloudiness, most likely Hurricane Ivan. The close match between storm tracks and spore pathways provides further evidence that clouds in storm systems can protect spores from UV radiation very effectively.

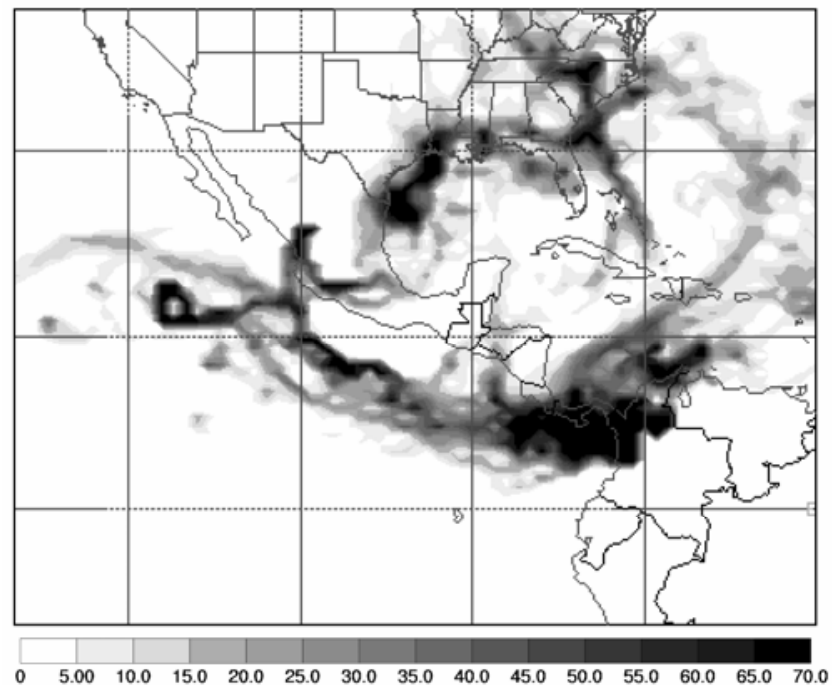

Fig. 6. Hindcast trajectory frequency at 10-m height during the 40-day period starting 7 August 2004. The high frequency represents preferred pathways using low source intensity ( 9 source points).
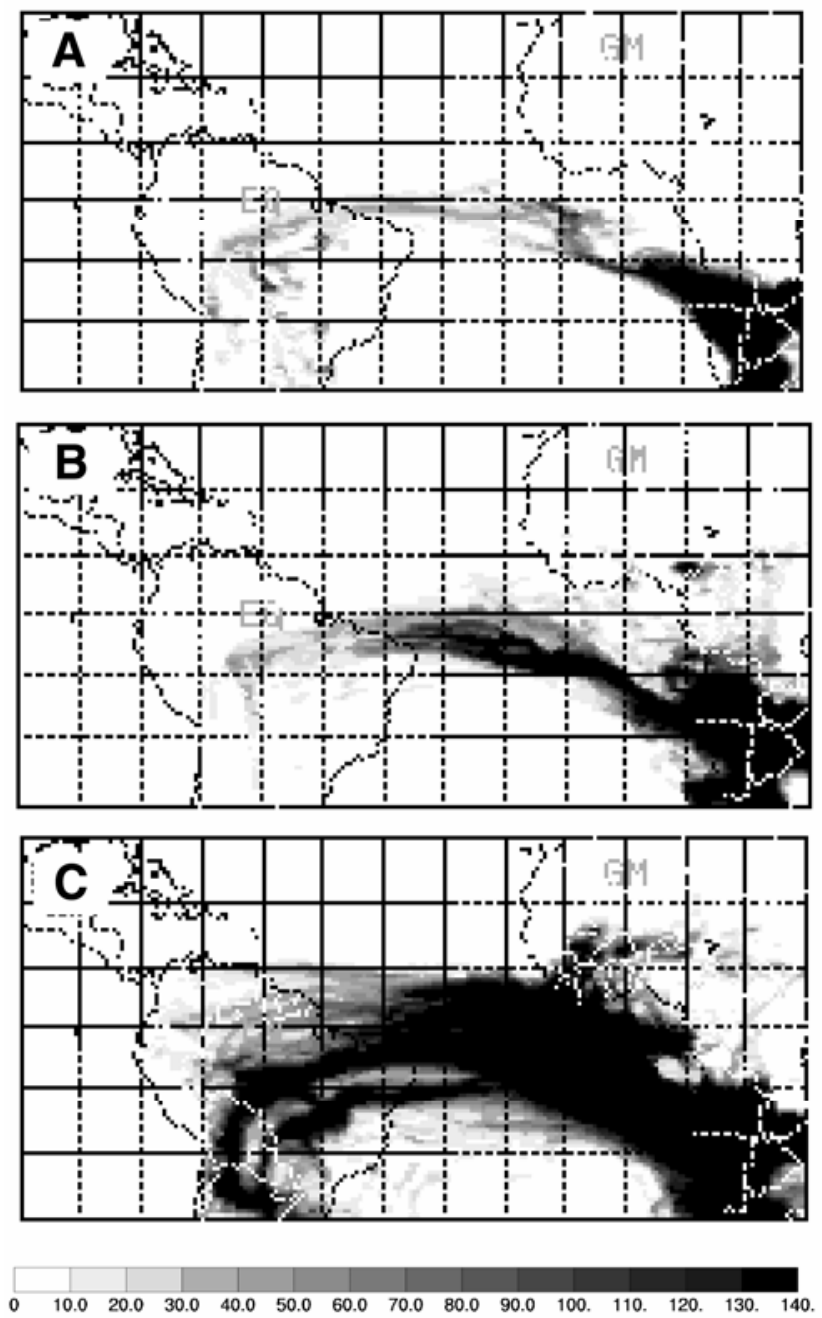

Fig. 7. Simulated monthly mean favorable pathways of soybean rust spore transport from Africa to South America during the first 3 months of 2001. The high frequency represents preferred pathways. A, January. B, February. C, March.

As a way of validating the model prediction, we used observed weather rather than the ECPC global prediction to drive our model (i.e., MM5) for the same 40-day period. The result showed clearly the concentrated trajectory frequency along the coastal states (Fig. 6). This hindcast is in close agreement with the corresponding forecasts, further demonstrating the reliability of long-term prediction.

Hurricane tracks and precipitation. Heavy precipitation associated with the hurricanes was another key factor in the rust invasion. Rainfall washes spores (through wet deposition) onto the ground and promotes inoculation of the rust $(26,29)$. For example, the heavy rainfall center in the vicinity of the eye wall of Ivan (to a lesser degree, Bonnie) coincided with $P$. pachyrhizi infection occurrence. This result strongly suggests the rainfall washout played a key role during the event. The heavy rainfall not only washed out the airborne spores but also provided moisture for subsequent disease development, as observed in other countries. Rainfall amount and frequency are very important determinants for the disease epidemics (26).

Other cases of validations. Using historical meteorological data, we hindcast favorable pathways originating from known sources in northeastern Zimbabwe and southeastern Zambia in Africa (7) for the 1998 to 2002 period during which the rust was transported to South America. Among the 5 years simulated, 2001 had most favorable pathways as shown by the connected trajectories across the Atlantic Ocean and extending southward to the Paraguay region (Fig. 7), in agreement with the first SBR detection in South America in 2001 (30). For the remaining 4 years, the conditions were not as favorable as 2001 (not shown).

Starting from the beginning of 2004, we have periodically predicted spore movement out of central South America. The model run in purely predictive mode forecasted spore movement a month in advance with sources being located near the Paraguay-Argentina-Brazil border (7). The trajectories showed a distinct strand from central South America toward Cali, Columbia, during 15 March to 15 April 2004 (Fig. 8), consistent with the SBR disease report (16).

During the 2005 growing season, we ran our model on a weekly basis, predicting disease activity 2 to 4 weeks in advance. The source intensity and locations were updated based on confirmed disease sites posted by USDA/APHIS (27). As an example, the model prediction of spore concentration early in the growing season agrees reasonably well with the confirmed disease distribution later (Fig. 9). The winds in the atmospheric boundary during the 2005 growing season were weaker and less northward than climatology (18), 
which could partly explain why the rust did not spread widely in 2005 . The model appears to have captured this feature.

\section{DISCUSSION}

We integrated a regional climate prediction model (MM5) and a particle dispersal model (HYSPLIT_4) into an Asian soybean rust spore prediction model system that can predict, a month in advance, the spore movement. The application of the model system to known $P$. pachyrhizi dispersal cases demonstrated that the model predicted correctly the spore movements from southern South America to Columbia and then to the United States in 2004, and the hindcast of the rust transport from Africa to South America also reflected this prediction. The quasi-operational prediction in the 2005 growing season further indicated that the model can capture the general pattern of disease spread in the southeastern states.

The month-long prediction of spore movement was based on the emerging seasonal forecast techniques that predict mean conditions (or climate), rather than day-to-day weather. Atmospheric predictability theory indicates that the day-to-day weather forecasts degrade to noise beyond about 2 to 3 weeks without external forcing (11). However, with strong sea surface temperature (SST) forcing over oceans and soil moisture over land as lower boundary conditions, the mean of atmospheric conditions can be predicted well beyond the 2 to 3 weeks. This extended prediction is based on slowly evolving lower boundary conditions and is referred to as "predictions of the second kind", compared with "predictions of the first kind" for daily weather forecasts based on initial conditions. The tropics are more responsive to the longlasting SST anomalies than middle latitudes. Thus, the potential predictability for the tropics at long-time scales is greater than that of extratropics (17), and the atmospheric predictability chiefly remains in oceans because of their large heat capacity. Successful forecasting of 1997-98 El Niño is an encouraging example of long-term seasonal forecasts (2). Thus, spore movement prediction over a month is built on the framework of climate prediction. It should be cautioned, however, that any long-term prediction has inevitable uncertainty to it. Reliable prediction of airborne spore movement heavily depends on meteorological prediction by MM5 in this study. The MM5 has been validated extensively elsewhere because of its wide application and thus was not validated thoroughly in the current effort.

Since climate forecasts represent only the average conditions over a period, the exact timing of spore arrival is not very meaningful. Thus, it was not our intention to overly emphasize the timing of spore arrival. Nevertheless, dated analysis of the single release of spores seemed to suggest that the western strand occurred in early August and is likely responsible for the rust in LA and neighboring states, while the eastern one occurred in early to middle

September and is likely responsible for the disease in GA and FL. The earlier spore track was possibly associated with tropical storm Bonnie, while the later one was

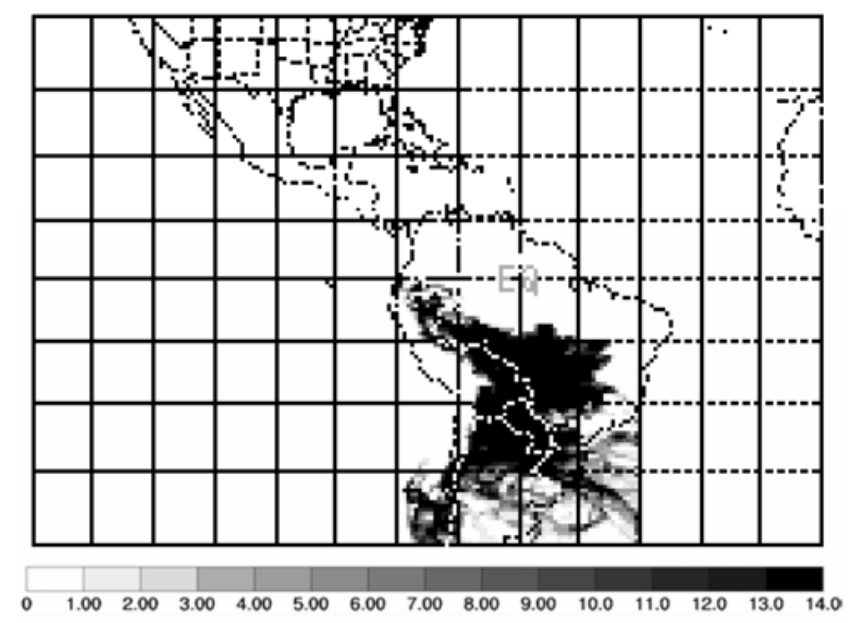

Fig. 8. Predicted favorable spore pathways based on 15 March 2004 weather conditions, showing a preferable pathway extending from southern South America toward Cali, Columbia. The trajectory frequency is averaged over the 15 March to 15 April period. The high frequency represents preferred pathways.
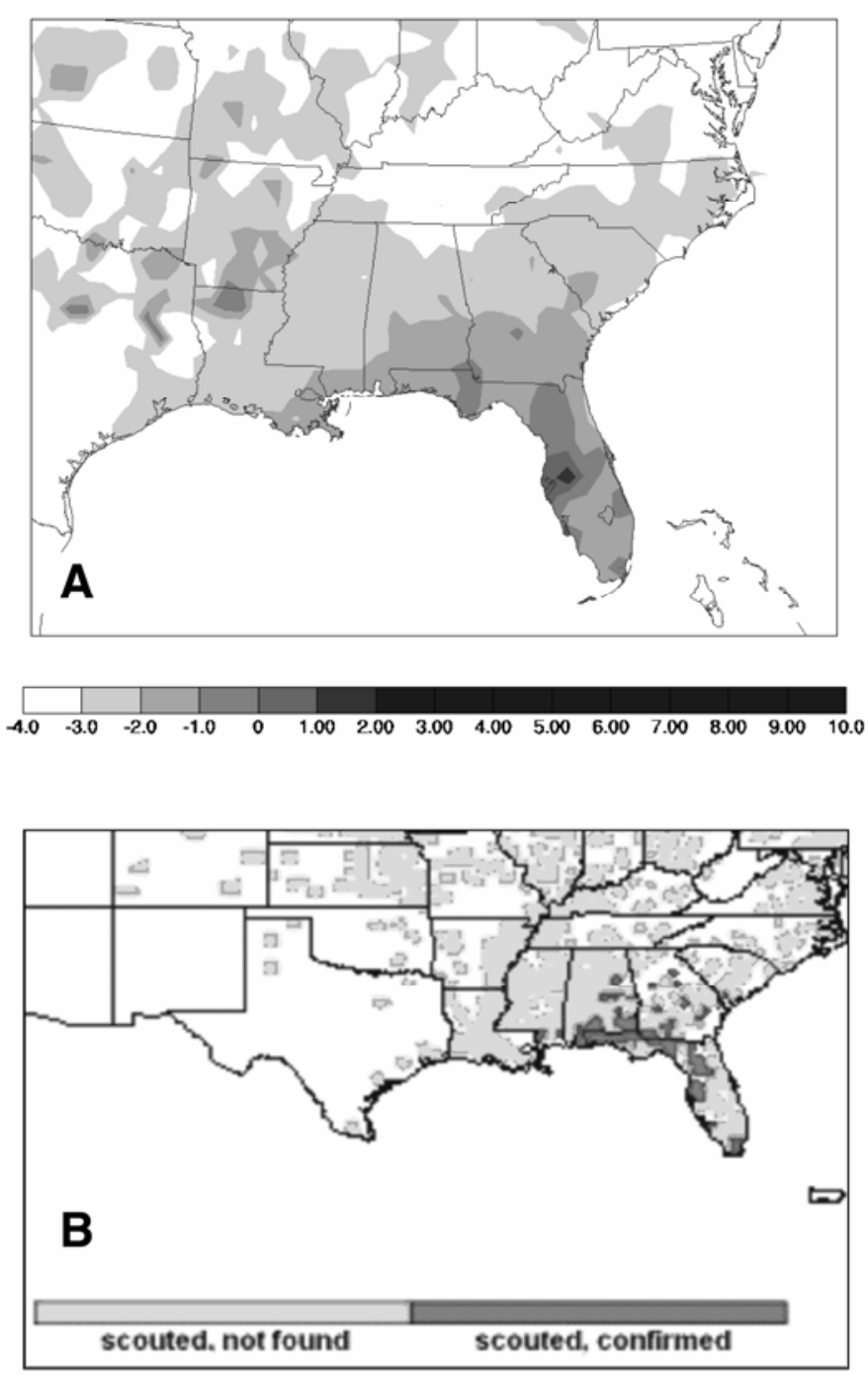

Fig. 9. Comparison of model-predicted spore concentration with rust disease distribution. A, Predicted spore concentration at 10-m height accumulated from 7 May to 23 July 2005. B, USDA-confirmed rust map as of 31 August 2005 
likely related to Hurricane Ivan. The less meaningful timing of events in the model probably explains why the time difference in the two strands (about 1 month) does not exactly correspond to the time difference of a few days in disease occurrence among states. Resolving timing inconsistency requires short-term hindcast simulations and warrants further research in the future.

The validation of spore prediction is extremely difficult because of the lack of precise observations of $P$. pachyrhizi spores. In this study, the model prediction was validated based on the rust disease activity that depends on other environmental conditions, aside from spore variability. Thus, using disease spread to validate spore or trajectory prediction can be viewed as a necessary condition. In other words, disease detection indicates the deposited spores, but spore existence does not necessarily mean disease occurrence. As observed $P$. pachyrhizi spore data may become available in the coming years, we would be able to validate the model against spore concentration and hopefully to improve model formulations. One such improvement will be the refinement of source determination and viability functions, which were crudely specified in the present study. Since the rust is expected to cause considerable yield loss to U.S. soybean production in coming years if major epidemics occur, our model prediction can serve as a useful guide for early warning and detection of the disease. Furthermore, in principle, the model also can be used for predicting other airborne plant diseases.

\section{ACKNOWLEDGMENTS}

We thank Roland Draxeler for his help with the HYSPLIT 4 model. We are grateful to Jack Ritchie of ECPC of Scripps Institution of Oceanography for his support with the global model outputs. Major funds for the project were provided by the United Soybean Board, supplemented by the National Institute for Global Environmental Change (NIGEC) Great Plains Regional Center. We appreciate reviewers' and editor's critical comments and constructive suggestions that enhanced this paper.

\section{LITERATURE CITED}

1. Anthes, R. A. 1990. Recent applications of the Penn State/NCAR Mesoscale Model to synop- tic, mesoscale, and climate studies. Bull. Am. Meteorol. Soc. 71:1610-1629.

2. Barsugli, J. J., Whitaker, J. S., Loughe, A., Sardeshmukh, P. D., and Toth, Z. 1999. The effects of the 1997-98 El Nino on individual large-scale weather events. Bull. Am. Meteorol. Soc. 80:1399-1411.

3. Draxler, R. R., and Hess, G. D. 1998. An overview of the HYSPLIT_4 modeling system of trajectories, dispersion and deposition. Aust. Meteorol. Mag. 47:295-308.

4. Dudhia, J., Gill, D., Manning, K., Wang, W., and Bruyere, C. 2002. PSU/NCAR Mesoscale Modeling System (MM5 version 3) tutorial class notes and user's guide. National Center for Atmospheric Research, Boulder, CO. Published online.

5. Grell, G. A., Dudhia, J. F., and Stauffer, D. 1994. A description of the fifth generation Penn State/NCAR Mesoscale Model (MM5). NCAR Tech. Note, NCAR/TN-398+IA. National Center for Atmospheric Research, Boulder, CO. Published online.

6. Isard, S. A., Gage, S. H., Comtois, P., and Russo, J. M. 2005. Principles of the atmospheric pathway for invasive species applied to soybean rust. Bioscience 55:851-861.

7. Isard, S. A., Main, C., Keever, T., Magarey, R., Redlin, S., and Russo, J. M. 2004. Weatherbased assessment of soybean rust threat to North America final report to APHIS, 15 July 2004. APHIS soybean rust report. Published online.

8. Kawuki, E., Adipala, E., and Tukamuhabwa, P. 2003. Yield loss associated with soya bean rust (Phakopsora pachyrhizi Syd.) in Uganda. J. Phytopathol. 151:7-12.

9. Killgore, E., Heu, R., and Gardner, D. E. 1994. First report of soybean rust in Hawaii. Plant Dis. 78:1216.

10. Kuchler, F., Duffy, M., Shrum, R. D., and Dowler, W. M. 1984. Potential economic consequences of the entry of an exotic fungal pest: The case of soybean rust. Phytopathology 74:916-920.

11. Lorenz, E. N. 1982. Atmospheric predictability experiments with a large numerical model. Tellus 34:505-513.

12. Main, C. E. 1996-2002. Blue mold historical forecasts; North American Plant Disease Forecast Center. Archives for Blue mold. North American Plant Disease Forecast Center. Published online.

13. Nagarajan, S., and Singh, D. V. 1990. Long distance dispersion of rust pathogens. Annu. Rev. Phytopathol. 28:139-153.

14. Nagarajan, S., Singh, D. V., Joshi, L. M., and Saari, E. E. 1976. Meteorological conditions associated with long-distance dissemination and deposition of Puccinia gramini tritici uredospores in India. Phytopathology 66:198-203.

15. National Oceanic and Atmospheric Admini- stration (NOAA). 2002. HYSPLIT User's Guide, NOAA Tech Memo ERL ARL-230, June 1999, 35 p. Last revised March 2002.

16. Northeast Plant Disease Diagnostic Network. 2004. Spotlight on Soybean Rust. October issue. Published online.

17. Palmer, T. N., and Andersom, L. T. 1994. The prospects for seasonal forecasting - A review. Quart. J. Roy. Meteorol. Soc. 120:755-793.

18. Pan, Z., Del Ponte, E., Xue, L., Li, X., Andrade, D., Pasken, R., and Yang, X. B. 2005. Soybean rust dispersal prediction and analysis in the U.S. for 2005 growing season. Preprint, National Soybean Rust Symposium, Nashville, TN, 15-16 November 2005.

19. Pan, Z., Pivonia, S., Pasken, R., Pietrowicz, J., and Yang, X. B. 2004. Simulation of airborne dispersal potentials of soybean rust from Africa to South America and from South America to North America. (Abstr.) Phytopathology 94:S80

20. Pasken, R., and Pietrowicz, J. A. 2005. Using dispersion and mesoscale meteorological models to forecast pollen concentrations. Atmos. Environ. 39:7689-7701.

21. Pivonia, S., Yang, X. B., and Pan, Z. 2005. Assessment of epidemic potential of soybean rust in the United States. Plant Dis. 89:678682.

22. Pretorius, Z. A., Kloppers, F. J., and Frederick, R. D. 2001. First report of soybean rust in South Africa. Plant Dis. 85:1288.

23. Reichler, T., and Roads, J. O. 2005. Longrange predictability in the tropics. Part I: Monthly averages. J. Climate 18:619-633.

24. Roads, J. O., Chen, S.-C., and Fujioka, F. 2001. ECPC's weekly to seasonal global forecasts. Bull. Am. Meteorol. Soc. 82:639-658.

25. Rotem, J., Wooding, B., and Aylor, D. E. 1985 The role of solar radiation, especially ultraviolet, in the mortality of fungal spores. Phytopathology 75:510-514.

26. Tan, Y. J., Yu, Z. L., and Yang, C. Y. 1996. Soybean Rust. China Agricultural Press, Beijing, China.

27. U.S. Dep. Agric. APHIS. 2004. USDA public soybean rust website published online through APHIS.

28. Yang, X. B., Dowler, W. M., and Royer, M. H. 1991. Assessing the risk and potential impact of an exotic plant disease. Plant Dis. 75:976982.

29. Yang, X. B., Royer, M. H., Tschanz, A. T., and Tsai, B. Y. 1990. Analysis and quantification of soybean rust epidemics from seventy-three sequential planting experiments. Phytopathology 80:1421-1427.

30. Yorinori, J. T., Pavia, W. M., Frederick, R. D., and Fernandez, P. F. T. 2002. Ferrugem da soja (P. pachyrhizi) no Brazsil e no Paraguai, nas safras 2000/01 e 2001/02. In II Congresso Brasileiro de Soja (Foz do Iguacu, PR). Embrapa Soja, Londrina, Paraguay. 